\title{
Feedback Signal from Motoneurons Influences a Rhythmic Pattern Generator
}

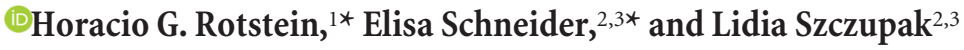 \\ ${ }^{1}$ Department of Mathematical Sciences, New Jersey Institute of Technology, University Heights, Newark, New Jersey 07102, ${ }^{2}$ Departamento de Fisiología, \\ Biología Molecular y Celular Dr. Héctor Maldonado, Universidad de Buenos Aires, Facultad de Ciencias Exactas y Naturales, and ${ }^{3}$ Instituto de Fisiología, \\ Biología Molecular y Neurociencias, CONICET-Universidad de Buenos Aires, 1428 Buenos Aires, Argentina
}

\begin{abstract}
Motoneurons are not mere output units of neuronal circuits that control motor behavior but participate in pattern generation. Research on the circuit that controls the crawling motor behavior in leeches indicated that motoneurons participate as modulators of this rhythmic motor pattern. Crawling results from successive bouts of elongation and contraction of the whole leech body. In the isolated segmental ganglia, dopamine can induce a rhythmic antiphasic activity of the motoneurons that control contraction (DE-3 motoneurons) and elongation (CV motoneurons). The study was performed in isolated ganglia where manipulation of the activity of specific motoneurons was performed in the course of fictive crawling (crawling). In this study, the membrane potential of CV was manipulated while crawling was monitored through the rhythmic activity of DE-3. Matching behavioral observations that show that elongation dominates the rhythmic pattern, the electrophysiological activity of $\mathrm{CV}$ motoneurons dominates the cycle. Brief excitation of $\mathrm{CV}$ motoneurons during crawling episodes resets the rhythmic activity of DE-3, indicating that CV feeds back to the rhythmic pattern generator. CV hyperpolarization accelerated the rhythm to an extent that depended on the magnitude of the cycle period, suggesting that CV exerted a positive feedback on the unit(s) of the pattern generator that controls the elongation phase. A simple computational model was implemented to test the consequences of such feedback. The simulations indicate that the duty cycle of $\mathrm{CV}$ depended on the strength of the positive feedback between $\mathrm{CV}$ and the pattern generator circuit.
\end{abstract}

Key words: duty cycle; dye coupling; leech crawling; left-right symmetry; Morris-Lecar model; phase relationship

\section{Significance Statement}

Rhythmic movements of animals are controlled by neuronal networks that have been conceived as hierarchical structures. At the basis of this hierarchy, we find the motoneurons, few neurons at the top control global aspects of the behavior (e.g., onset, duration); and within these two ends, specific neuronal circuits control the actual rhythmic pattern of movements. We have investigated whether motoneurons are limited to function as output units. Analysis of the network that controls crawling behavior in the leech has clearly indicated that motoneurons, in addition to controlling muscle activity, send signals to the pattern generator. Physiological and modeling studies on the role of specific motoneurons suggest that these feedback signals modulate the phase relationship of the rhythmic activity.

\section{Introduction}

The organization of networks that control rhythmic motor behaviors is markedly hierarchical: numerous motoneurons are in

\footnotetext{
Received March 13, 2017; revised Aug. 1, 2017; accepted Aug. 9, 2017.

Author contributions: L.S. designed research; H.G.R., E.S., and L.S. performed research; E.S. and L.S. analyzed data; L.S. wrote the paper.

This work was supported by Agencia Nacional de Promoción Científica y Tecnológica PICT 2012-2360 and Universidad de Buenos Aires Ciencia y Tecnología, University of Buenos Aires 20020150100179BA to L.S. We thank Drs. Martín Carbó-Tano, María Fernanda Ceriani, and Violeta Medan for highly valuable comments on the manuscript. The authors declare no competing financial interests.

*H.G.R. and E.S. contributed equally to this work.

Correspondence should be addressed to Dr. Lidia Szczupak, Ciudad Universitaria, Pabellón II, piso 2, FBMC-FCEN, 1428 Buenos Aires, Argentina. E-mail: szczupak@retina.ar.
}

charge of controlling muscle activity at the base of the hierarchy; and at the top, fewer neurons control the onset, duration, and other global aspects of behavior (Kristan et al., 2005; Goulding, 2009; Puhl et al., 2012; Drew and Marigold, 2015; Georgopoulos and Carpenter, 2015). Between these two ends, networks distributed throughout the nervous system determine the actual rhythmic pattern of movements, and thus they are recognized as pattern generators. Old and new studies have questioned whether motoneurons are solely output elements or whether they actively participate in pattern generation. Classical examples 
A

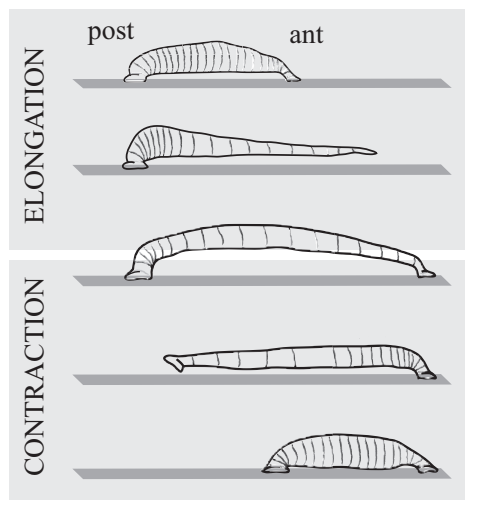

B

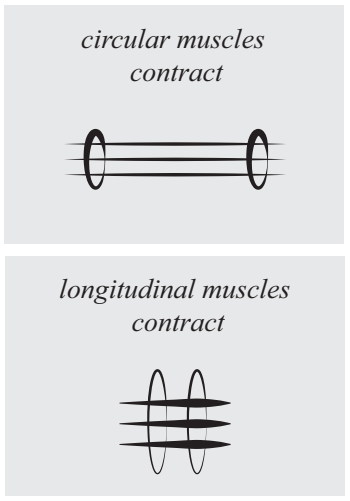

Figure 1. Crawling motor pattern. $\boldsymbol{A}$, The drawing represents a leech performing a crawling step. Crawling results from the rhythmic succession of elongation and contraction of the tubular body, anchored on the suckers located at the anterior (ant) and posterior (post) ends (adapted from Stern-Tomlinson et al., 1986). B, Schematic of muscle activity. Top, Elongation is caused by the activation of the circular muscles (represented as ovals). Bottom, Contraction by the activation of the longitudinal muscles (indicated as horizontal thick lines). During elongation, the segmental diameter decreases and the anteroposterior length is increased; during contraction, the length is reduced and the circumference is increased.

from invertebrates have established that motoneurons do indeed participate in central networks (Maynard and Selverston, 1975; Kristan and Calabrese, 1976; Friesen et al., 1978; Poon et al., 1978; Selverston and Moulins, 1985; Staras et al., 1998; Barth, 2000); and more recently, this has been found in a vertebrate system (Song et al., 2016). An important question yet to be addressed is what is the distinct functional contribution of motoneurons to pattern generation. Are they intrinsic elements of the pattern generator circuits or are they extrinsic modulators of these circuits?

The analysis of this question in any organism requires that motoneurons can be readily identified and that their activity can be both monitored and experimentally manipulated. The leech nervous system is particularly suited for such an approach (Wagenaar, 2015). Crawling is a rhythmic motor behavior by which leeches locomote on solid surfaces. This behavior results from successive bouts of elongation and contraction of the whole body, anchored on front and rear suckers (Fig. 1A). The elongation and contraction of the body are executed by the activation of circular and longitudinal muscles, respectively (Gray et al., 1938) (Fig. $1 B$ ). The motor pattern can be monitored in the isolated nervous system, through the activity of specific motoneurons by means of extra and intracellular recordings (Eisenhart et al., 2000), and can be effectively evoked by bath applied dopamine (Puhl and Mesce, 2008; Bernardo Perez-Etchegoyen et al., 2012). Throughout the text, we use italics (crawling) to refer to the motor pattern monitored in the isolated nervous system.

Here we have studied the role played by a pair of motoneurons that control the activity of the muscles responsible for the elongation phase of crawling, the circular ventral (CV) motoneurons. Matching the behavioral output, the activity of the CV motoneurons dominates the cycle. We found that experimental activation of these neurons reset the rhythm and their inhibition shortened the period of crawling. Physiological and modeling experiments suggest that a positive feedback between the $\mathrm{CV}$ motoneuron and the pattern generator circuit modulates the duration of the elongation phase.

\section{Materials and Methods}

Biological preparation. Leeches (Hirudo $\mathrm{sp}$ ) weighing 2-5 g, were obtained from commercial suppliers (Leeches USA and Niagara Leeches) and maintained at $15^{\circ} \mathrm{C}$ in artificial pond water. These animals are hermaphrodites. The leech nervous system is composed of a chain of 21 midbody ganglia in between head and tail brains. Each midbody ganglion contains all the sensory and motor neurons that innervate the corresponding segment (Muller et al., 1981).

Studies were performed in isolated single ganglia. The tissue was bathed in normal saline ( $115 \mathrm{~mm} \mathrm{NaCl}, 4 \mathrm{~mm} \mathrm{KCl}, 1.8 \mathrm{~mm} \mathrm{CaCl}_{2}, 1 \mathrm{~mm}$ $\mathrm{MgSO}_{4}, 10 \mathrm{~mm}$ HEPES, $10 \mathrm{~mm}$ glucose, $\mathrm{pH}$ 7.4), at room temperature $\left(20^{\circ} \mathrm{C}-25^{\circ} \mathrm{C}\right)$ and pinned to Sylgard (Dow Corning) in a recording chamber. The sheath covering the ganglion was dissected away, leaving the neuronal cell bodies exposed to the external solution.

Electrophysiological recordings. Intracellular somatic recordings were made with microelectrodes pulled from borosilicate capillary tubing (FHC), filled with $3 \mathrm{~m}$ potassium acetate (resistance $20-40 \mathrm{~m} \Omega$ ). The electrodes were connected to an Axoclamp 2B amplifier (Molecular Devices) operating in bridge mode or, when stated, in discontinuous current-clamp mode. Extracellular activity was recorded from dorsal posterior nerves using suction electrodes connected to a differential AC amplifier (Neuroprobe 1700, A-M Systems). The intracellular and extracellular recordings were digitized using an analog digital converter (Digidata 1440, Molecular Devices) and acquired using a commercial program (Clampex 9.2, Molecular Devices) at a sampling rate of $5 \mathrm{kHz}$. The studied neurons are readily recognized by their soma location, electrophysiological properties, and synaptic connectivity.

To evoke crawling, the ganglion was continuously superfused with $75 \mu \mathrm{M}$ dopamine (Puhl and Mesce, 2008). The dopamine solution was prepared fresh at the beginning of each experimental day, and only one crawling episode was evoked per ganglion.

Histological procedures. Identified neurons were impaled with an intracellular electrode whose tip was filled with rhodamine or Texas Redconjugated dextran (MW $3 \mathrm{kDa}$, Invitrogen) or Neurobiotin (Vector Laboratories). The markers were loaded into the cells by iontophoresis using microelectrodes loaded with a $5 \%$ solution of the dye; positive 2-4 nA pulses ( $500 \mathrm{~ms}, 1 \mathrm{~Hz}$ ) were injected for 10-20 min and let diffuse for at least an hour. Ganglia were fixed with $4 \%$ PFA. To reveal the presence of Neurobiotin, the fixed ganglia were incubated with $50 \mu \mathrm{g} / \mathrm{ml}$ AlexaFluor-488 streptavidin D (Vector Laboratories) in 1\% Triton $\mathrm{X}-100$ phosphate buffer for $2 \mathrm{~h}$ at room temperature; and then washed in phosphate saline, dehydrated, cleared in methyl salicylate, and mounted in DePex (E.M.S.). The fluorescent images were examined using a 488 $\mathrm{nm}$ Argon or a $543 \mathrm{~nm}$ HeNe laser line of an Olympus FLUOVIEW FV300 confocal system with a $20 \times$ objective.

Data analysis. Data analysis was performed using commercial software (Clampfit 9, Molecular Devices and Axograph 1.5.4). To generate the voltage-current curves shown in Figure 3, we measured the membrane potential $\left(V_{\mathrm{m}}\right)$ as the mean $V_{\mathrm{m}}$ during the last $200 \mathrm{~ms}$ of the pulse. Spikes were detected using amplitude threshold after applying a high-pass filter to the recordings, mean frequency was calculated as number of spikes in a time interval over the interval duration, and instantaneous firing frequency was calculated as the reciprocal of the time between two successive spikes.

Crawling was characterized by the cycle period, measured as the time elapsed between the first DE-3 spikes in two successive bursts: duration of bursts, measured as the time between the first and last spike in a burst; and the firing frequency, calculated as the number of spikes in a burst divided by the burst duration. Duty cycle is calculated as the ratio between the duration of a burst over the corresponding cycle period.

Unless otherwise stated, data are presented as mean \pm SEM. Curve fitting was achieved using commercial software (Kaleidagraph 3.0.2, Abelbeck Software).

Biophysical (conductance-based) network model. The biophysical (conductance-based) network model we used consists of four cells: C (contraction), E (elongation), DE-3, and CV. Cells C and E were modeled using the Morris-Lecar equations (Morris and Lecar, 1981; Rinzel and Ermentrout, 1998), whereas cells DE-3 and CV were modeled using the 
A
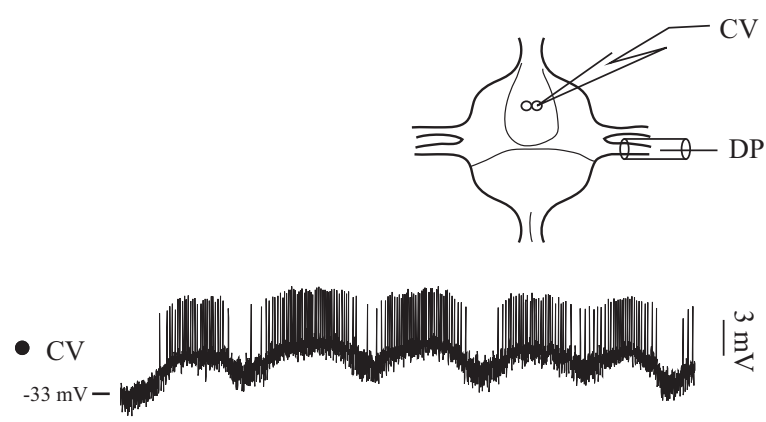

- DP
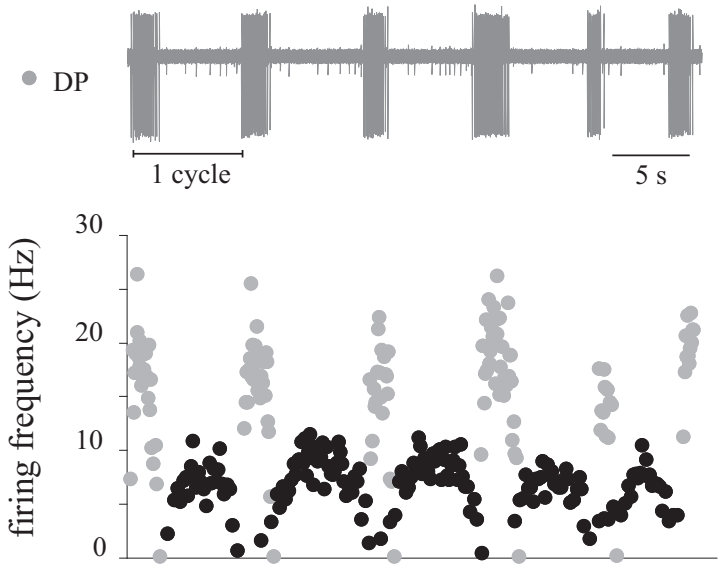

B

C
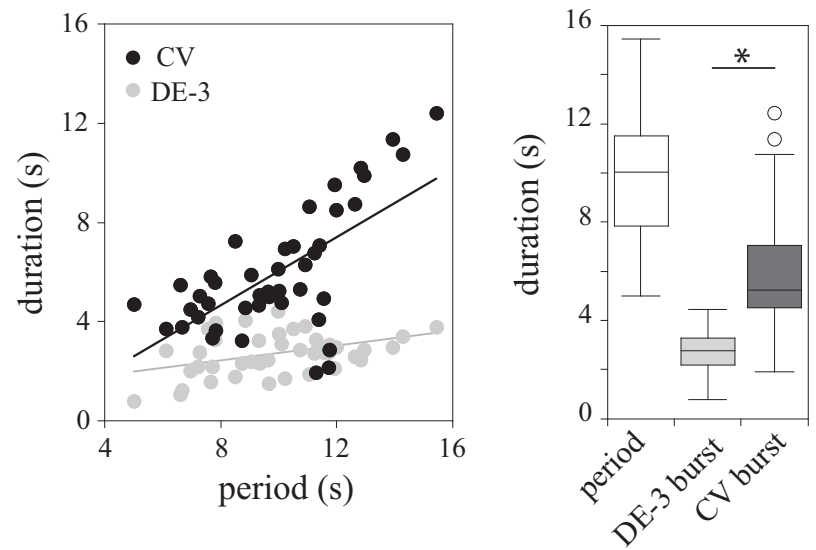

D

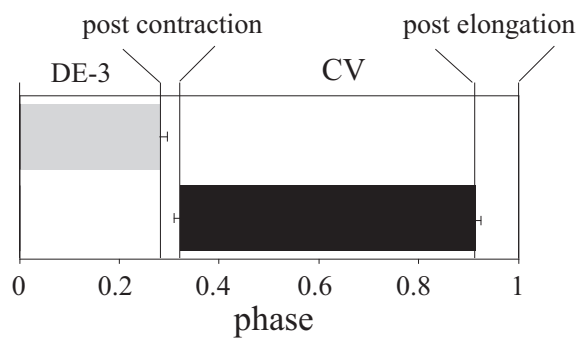

Figure 2. CV and DE-3 activity during crawling. $A$, Top right, Diagram represents the recording configuration: intracellular recording of a CV motoneuron (top trace) and extracellular recording of a DE-3 neuron in the DP nerve (bottom gray trace) performed in an isolated ganglion during a dopamine-induced crawling episode. Left, The membrane potential of the CV. Bottom, Graph of the recordings represents the instantaneous firing frequency of CV (black dots) and DE-3 motoneurons (gray dots) in the same temporal scale as the recordings. $\boldsymbol{B}$, Burst duration of $\mathrm{CV}$ and DE-3 as a function of the cycle period. The lines show the linear fit: for $\mathrm{DE}-3, r=0.4$, slope $=0.15 \pm 0.05$, and $p=0.0063$; for $\mathrm{CV}, r=0.65$, slope $=0.69 \pm 0.12$, and $p=$ 0.0000022 . C, Box plot represents the cycle period, and the duration of DE-3 and CV bursts. passive membrane equation (no active ionic currents). Cells $\mathrm{C}$ and $\mathrm{E}$ are connected through recurrent $\mathrm{GABA}_{\mathrm{A}}$-like inhibition and project to $\mathrm{DE}-3$ and $C V$, respectively, through AMPA-like excitation. When the feedback connection from $\mathrm{CV}$ to $\mathrm{E}$ is included, an AMPA-like excitation simulates the connection between these two units.

The current-balance equation for the Morris-Lecar model we used for cells $\mathrm{C}$ and $\mathrm{E}$ has the following form:

$$
C \frac{d v}{d t}=I_{a p p}-G_{L}\left(v-E_{L}\right)-G_{C a} m_{\infty}(v)\left(v-E_{C a}\right)-G_{K} w\left(v-E_{K}\right),
$$

where $v$ is the membrane potential $(\mathrm{mV}), t$ is time $(\mathrm{ms}), C$ is the membrane capacitance, $I_{a p p}$ is the applied bias (DC) current, $G_{L}\left(v-E_{L}\right)$ is the leak current, $G_{C a} m_{\infty}(v)\left(v-E_{C a}\right)$ is a $\mathrm{Ca}^{2+}$ current with a fast-activation variable $m_{\infty}(v)$ slave to voltage, and $G_{K} w\left(v-E_{K}\right)$ is a noninactivating $\mathrm{K}^{+}$ current with activation gating variable $w . G_{L}, G_{C a}$ and $G_{K}$ are maximal conductances, and the parameters $E_{L}, E_{C a}$, and $E_{K}$ are the reversal potentials of these currents. The gating variable $w$ obeys the following differential equation:

$$
\frac{d w}{d x}=\phi \frac{w_{\infty}(v)-w}{\tau_{w}(v)}
$$

where $w_{\infty}(v)$ and $\tau_{w}(v)$ are the voltage-dependent activation curve and time constant, respectively. The parameter $\phi$ determines the rate of change of variable $w$ relative to the voltage. The functions $m_{\infty}(v), w_{\infty}(v)$, and $\tau_{w}(v)$ are given by the following:

$$
\begin{aligned}
& m_{\infty}(v)=\frac{1}{2}\left(1+\tanh \frac{v-V_{1}}{V_{2}}\right) \\
& w_{\infty}(v)=\frac{1}{2}\left(1+\tanh \frac{v-V_{5}}{V_{6}}\right) \\
& \tau_{w}(v)=\frac{1}{2}\left(1+\cosh \frac{v-V_{3}}{V_{4}}\right)^{-1}
\end{aligned}
$$

We used the following parameter values: $C=20 \mu \mathrm{F} / \mathrm{cm}^{2}$, Iapp $=0.80$ $\mu \mathrm{A} / \mathrm{cm}^{2}, G_{L}=0.020 \mathrm{mS} / \mathrm{cm}^{2}, G_{C a}=0.044 \mathrm{mS} / \mathrm{cm}^{2}, G_{K}=0.060 \mathrm{mS} / \mathrm{cm}^{2}$, $E_{L}=-60 \mathrm{mV}, E_{C a}=120 \mathrm{mV}, E_{K}=-84 \mathrm{mV}, V_{1}=-1.2 \mathrm{mV}, V_{2}=25$ $\mathrm{mV}, V_{3}=2 \mathrm{mV}, V_{4}=30 \mathrm{mV}, V_{5}=2 \mathrm{mV}$, and $V_{6}=30 \mathrm{mV}$.

The motoneurons $\mathrm{C}$ and $\mathrm{E}$ were modeled using current-balance equation similar to that for the Morris-Lecar model, but excluding the $\mathrm{Ca}^{2+}$ and $\mathrm{K}^{+}$currents. We used the following parameter values: $C=20 \mu \mathrm{F} / \mathrm{cm}^{2}$, Iapp $=0 \mu \mathrm{A} / \mathrm{cm}^{2}, G_{L}=0.010 \mathrm{mS} / \mathrm{cm}^{2}$, and $E_{L}=-60 \mathrm{mV}$.

The excitatory (AMPA-like) and inhibitory $\left(\mathrm{GABA}_{\mathrm{A}}\right.$-like) synaptic currents we used are described by the following:

$$
I_{s y n}=-G_{s y n} S_{p r e}\left(V_{p o s t}-E_{s y n}\right)
$$

where $G_{s y n, X Y}\left(G_{e x, X Y}\right.$ for AMPA and $G_{i n, X Y}$ for $\left.\mathrm{GABA}_{\mathrm{A}}\right)$ is the maximal synaptic conductance from a presynaptic neuron $X$ to a postsynaptic neuron $Y$ (in $\mathrm{mS} / \mathrm{cm}^{2}, \mathrm{G}_{\mathrm{in}, 12}=0.01, \mathrm{G}_{\mathrm{in}, 21}=0.01, \mathrm{G}_{\mathrm{ex}, 23}=0.02$ and $\mathrm{G}_{\mathrm{ex}, 14}=0.02, \mathrm{G}_{\mathrm{ex}, 32}=2.6$ where cell 1 is $\mathrm{C}$, cell 2 is $\mathrm{E}$, cell 3 is $\mathrm{CV}$, and cell 4 is DE-3), $S_{\text {pre }}$ is the presynaptic function (depending on the voltage of the presynaptic neuron among other factors) describing the fraction of open synaptic channels and $E_{s y n}$ is the synaptic reversal potential $\left(E_{s y n}=\right.$ $20 \mathrm{mV}$ for AMPA and $-60 \mathrm{mV}$ for $\mathrm{GABA}_{\mathrm{A}}$ ). For each connection from a presynaptic neuron $\mathrm{X}$ to a postsynaptic neuron $\mathrm{Y}$ in the network, we added a term of the form $I_{s y n}$ to the current balance equation for the neuron Y. The dynamics of $S_{\text {pre }}$ (dependent on the dynamic of X) obeys a kinetic equation of the following form:

$\leftarrow$

${ }^{*} p<0.0001$ (Wilcoxon test for paired data). $\boldsymbol{D}$, Horizontal bars represent the average ( \pm SEM) timing at which the bursts of DE- 3 and $C V$ began and ended, relative to the duration of the period. Two additional phases are indicated: (1) between the end of $D E-3$ burst and the beginning of $\mathrm{CV}$ burst (after contraction); and (2) between the end of $\mathrm{CV}$ burst and the beginning of the next cycle (after elongation). $\boldsymbol{B}-\boldsymbol{D}, n=43$ cycles/10 crawling episodes in 8 different animals. 


$$
\frac{d S_{\text {pre }}}{d x}=N\left(v_{\text {pre }}\right) \frac{1-S_{\text {pre }}}{\tau_{\text {rise }}}-\frac{S_{\text {pre }}}{\tau_{\text {decay }}}
$$

where:

$$
N(v)=\frac{1}{2}\left[1+\tanh \left(\frac{v-2}{5}\right)\right]
$$

and $\tau_{\text {rise }}$ and $\tau_{\text {decay }}$ are the rise and decay times, respectively, of excitation and inhibition. For AMPA-like excitation, we used $\tau_{\text {rise }}=1 \mathrm{~ms}$ and $\tau_{\text {decay }}=5 \mathrm{~ms}$. For $\mathrm{GABA}_{\mathrm{A}}$-like inhibition, we used $\tau_{\text {rise }}=0.2 \mathrm{~ms}$ and $\tau_{\text {decay }}=10 \mathrm{~ms}$.

\section{Results}

\section{CV activity during crawling}

CV motoneurons activate ventral circular muscle fibers that participate in the elongation phase of crawling, whereas DE-3 motoneurons activate longitudinal fibers and participate in the contraction phase (Stuart, 1970; Eisenhart et al., 2000). We have investigated the role of $\mathrm{CV}$ motoneurons in dopamine-induced crawling, as we monitored the performance of the motor pattern through the activity of DE-3 motoneurons. The work was performed in isolated midbody ganglia, as this is the minimal anatomical unit of the leech nervous system that expresses all the circuit elements necessary to generate this motor pattern (Puhl and Mesce, 2008; Bernardo Perez-Etchegoyen et al., 2012).

Simultaneous intracellular recordings of $\mathrm{CV}$ and extracellular recordings of DE-3 (the largest unit in DP nerves) motoneurons show that these two cells are active in antiphase (Fig. 2A). A plot describing the duration of the bursts of activity of each motoneuron as a function of the cycle period indicates that there is a linear correlation between the two variables (Fig. 2B). The CV bursts were more prolonged (Fig. 2C) and exhibited a steeper duration versus period slope than those of DE-3 (Fig. 2B).

Figure $2 D$ describes the average phase relationship of the two motoneurons within a cycle period, showing that the $\mathrm{CV}$ phase dominates the cycle.

\section{Physiological properties of CV motoneurons}

To evaluate the role played by CV in the motor pattern, we first investigated the intrinsic properties of this cell. The somata of this bilateral pair of motoneurons are located on the ventral face of each midbody ganglion, in the anterior medial packet. Figure $3 \mathrm{~A}$ illustrates that as most motoneurons in the leech, CV is monopolar and extends its major branch to the contralateral hemiganglion, giving rise to neuritic arborizations in the ipsilateral and contralateral neuropil. The axon exits the ganglion toward the periphery through the contralateral anterior root.

To examine the electrophysiological properties of $\mathrm{CV}$, we injected a series of current pulses (from -0.5 to $0.5 \mathrm{nA}$, at $0.1 \mathrm{nA}$ steps) and recorded the resulting changes in membrane potential
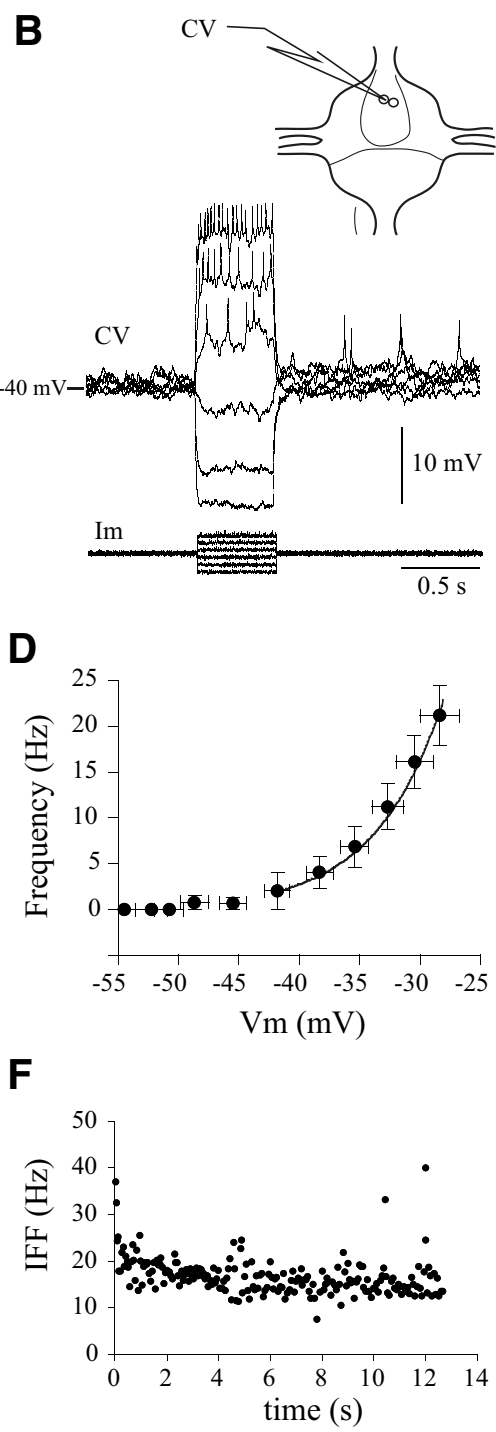

Figure 3. Properties of CV motoneurons. $A, C V$ anatomy obtained by filling the neuron with rhodamine-conjugated dextran. Confocal micrograph from a z stack of $1081 \mu \mathrm{m}$ optical sections. Dashed lines indicate the approximate margins of the midbody ganglion and the longitudinal midline. Anterior (ant) and posterior (post) connective nerves are indicated. $\boldsymbol{B}$, Diagram represents $-0.3,-0.1,0.1,0.3$, and $0.5 \mathrm{nA})$ in discontinuous current-clamp configuration (Im, bottom traces). In somatic recordings, the ion potentials are attenuated versions of signals initiated at an electrically distant region. $C$, Mean $V_{m}$ of $C V$ as a function of 政 a $13 \mathrm{~s}$ current pulse $(0.5 \mathrm{nA})$. Inset, Magnification of the recording segment enclosed in the line box. $\boldsymbol{F}$, Instantaneous firing frequency (IFF) in the recording shown in $\boldsymbol{E}$ as a function of time ( $t=0$ at the beginning of the pulse).

(Fig. 3B). The protocol shifted CV membrane potential between -60 and $-30 \mathrm{mV}$ and, in this range, the voltage-current relationship of the motoneurons fit a line (Fig. 3C) whose slope indicates a stable input resistance of $\sim 30 \mathrm{M} \Omega$. CV firing threshold was $\sim-40 \mathrm{mV}$, and its firing frequency fit an exponential function of CV membrane potential (Fig. 3D). When stimulated with a $13 \mathrm{~s}$ pulse CV fired at a nearly steady rate for the entire depolarization period (Fig. 3E,F).

Together with previous work (Bernardo Perez-Etchegoyen et al., 2012), the results suggest that the membrane potential envelope (ignoring the spikes) displayed by the motoneurons during crawling is ruled by their passive properties.

Several motoneurons in the leech are electrically coupled to their contralateral homolog (Ort et al., 1974; Fan et al., 2005). We 
A
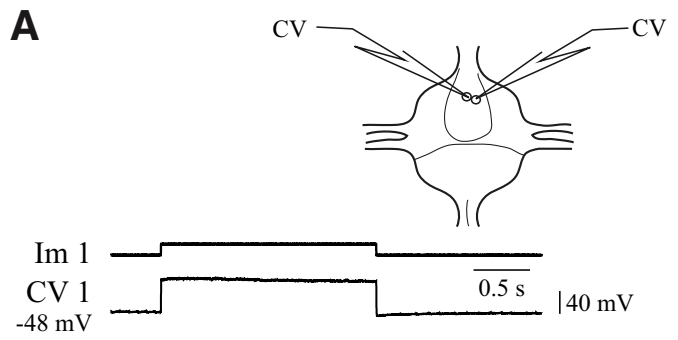

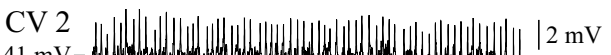

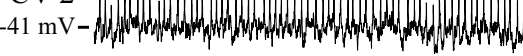

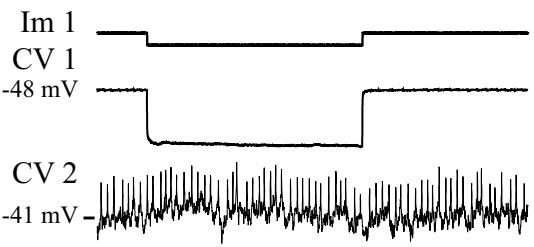

$\mathrm{Bi}$

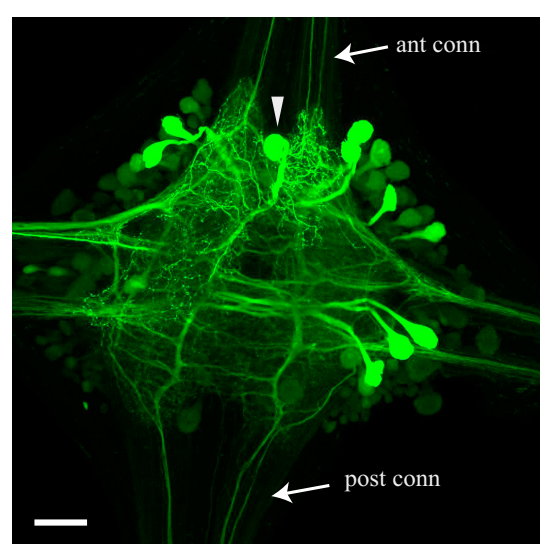

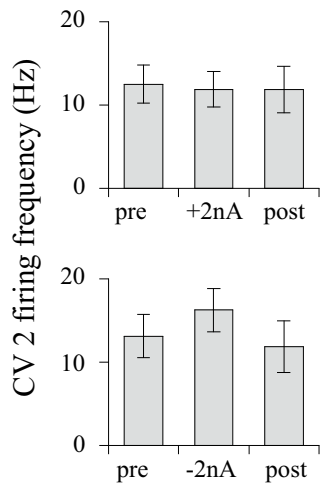

Bii

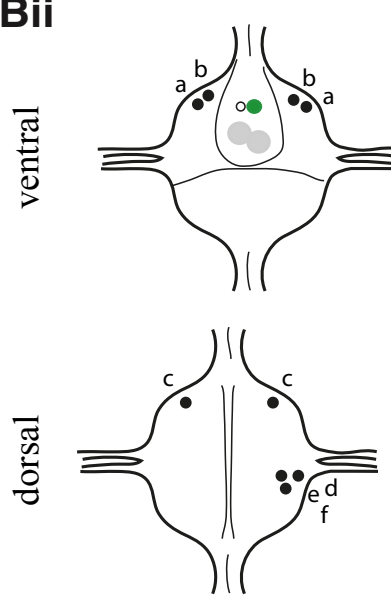

Figure 4. Electrical connections of CV motoneurons. $A$, Top diagram represents the recording configuration: simultaneous intracellular recordings of both CV motoneurons. Square current pulses were injected in one $C V$ while the $V_{m}$ of both neurons was recorded. The data are shown in two series of three traces each. From top to bottom, Current injected in CV1 (Im1), $V_{m}$ recordings of $\mathrm{CV} 1$ and $\mathrm{CV} 2$. Note the difference in the $V_{\mathrm{m}}$ scale of the two $\mathrm{CV}$ s. The top series corresponds to the response to $2 \mathrm{nA}$. The bottom series corresponds to $-2 \mathrm{nA}$. To the right of each series, the bars represent the corresponding mean ( $\pm S E M$ ) firing frequency before (pre), during, and after (post) the pulse ( $n=3$ ganglia from 2 animals). Bi, Confocal micrograph of a midbody ganglion where one CV neuron was loaded with Neurobiotin; the image results from the maximal intensity projections of $761 \mu \mathrm{m}$ optical sections through the $z$ axis. White triangle represents the $C V$ neuron that was loaded with Neurobiotin. Scale bar, $100 \mu \mathrm{m}$. Arrows indicate the anterior and posterior connectives. Bii, Drawings represent the approximate location of the neurons coupled to the injected CV. As a reference, large gray circles represent the position of the large Retzius neurons on the ventral surface and, anterior to them, the CV neurons. Green circle represents the CV loaded with Neurobiotin. White circle represents the contralateral homolog. Black circles represent the approximate position of the neurons dye-coupled to the Neurobiotin-loaded CV on the ventral (top) and dorsal (bottom) surfaces of the ganglia ( $n=9$ ganglia from 5 animals) in the majority of the preparations (see quantitative summary in Results). For further discussion, these soma are identified by letters a-f outside the ganglion profile.

tested electrical coupling between the pair of $\mathrm{CV}$ neurons by injecting current in one cell and measuring the resulting $V_{\mathrm{m}}$ change in both CVs. Figure $4 A$ illustrates that current pulses that produced large membrane potential changes in the injected cell did not cause detectable changes in the membrane potential and firing frequency in the contralateral cell. These results indicate that the bilateral pair of CVs is not electrically coupled.

\section{$\mathrm{CV}$ neurons are dye-coupled to several neurons in the ganglion}

The cell tracer Neurobiotin diffuses between electrically coupled neurons in the leech (Fan et al., 2005). Figure $4 B i$ shows that

injection of Neurobiotin in one CV neuron stained another 9 neurons in the ganglion. These 9 cells are distributed in the ventral and dorsal surfaces of the ganglion and were found consistently in specific locations, as depicted in Figure 4Bii: on the ventral surface, we found (in 7 of 9 ganglia) two bilateral pairs of cells in the anterior lateral packets (cells a and b); at the dorsal face, we found ( 7 of 9) one bilateral pair of cells located in the anterior packets (cell c) and (8 of 9) three cells in the posterior ipsilateral packet (cells d, e, and f).

Location and general morphological features are indicative of cellular identity in leech ganglia. As stated earlier, and shown for CV (Fig. 3A), motoneurons characteristically project their main branch to the periphery through contralateral roots and do not extend neurites through the connectives (Stuart, 1970; Poon et al., 1978; Fan et al., 2005). On these grounds, the neurons stained at the dorsal posterior packet (Fig. 4B; cells d, e, and f) are likely to be motoneurons. We hypothesized that these cells are inhibitors of longitudinal muscles, cells ventral inhibitor 2 (VI-2) and dorsal inhibitor 1 (DI-1) (Fan et al., 2005). A direct test of electrical coupling is very hard to achieve as the somata of $\mathrm{CV}$, and these inhibitory motoneurons are on the ventral and dorsal surfaces of the ganglion, respectively. We thus proceeded to identify DI-1 and VI-2 with the ganglion dorsal side up, and labeled them with rhodamine-conjugated dextran, and then turned the ganglion ventral side up and injected CV with Neurobiotin to proceed with the dye-coupling experiment as described in Figure $4 B$. The results clearly indicated that $\mathrm{CV}$ is not coupled to DI-1 (Fig. 5A) or VI-2 (Fig. 5B) as neither cells $\mathrm{d}$, nor $\mathrm{e}$, nor $\mathrm{f}$ coincided with the rhodamine-labeled motoneuron. The identity of cells $d$, e, or f remains to be determined.

The observation of neurites extending through the anterior and posterior connectives suggests that, in addition, the $\mathrm{CV}$ motoneurons are coupled to neurons that are not motoneurons. Possible candidates are the NS neurons (Wadepuhl, 1989; Rela and Szczupak, 2007), a pair of nonspiking premotor neurons whose somata are located in the position of cells a or b (Fig. 4Bii) and are electrically coupled to CV. Staining NS with rhodamine-conjugated dextran confirmed that cell $\mathrm{a}$ or $\mathrm{b}$ in Figure 4Bii is indeed the NS neuron (Fig. 5C).

Together, the results indicate that CV motoneurons are electrically coupled to at least nine neurons, among which two are the pair of premotor NS neurons. No soma was stained in the location where the contralateral CV neuron is expected, reinforcing the notion that the pair of CV motoneurons are not electrically coupled. 
A

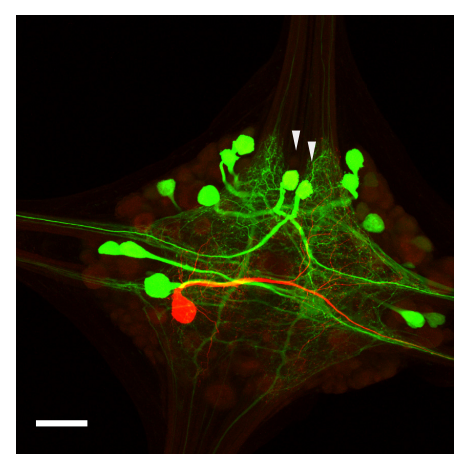

B

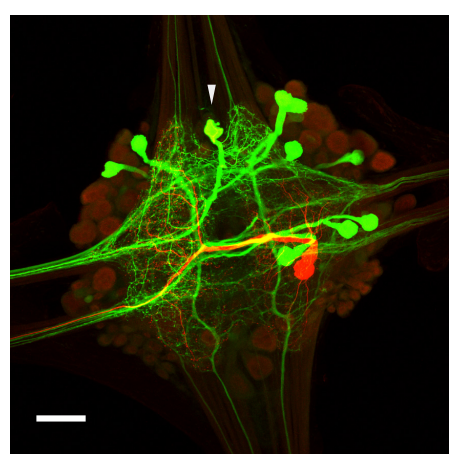

C

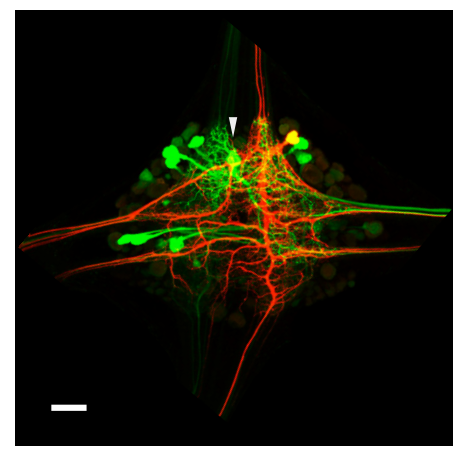

Figure 5. Dye coupling analysis of CV connectivity. $\boldsymbol{A}$, Confocal micrograph of a midbody ganglion (anterior up) where both CV neurons were loaded with Neurobiotin and a DI-1 neuron was loaded with rhodamine-conjugated dextran; the image results from the maximal intensity projections of $941 \mu \mathrm{m}$ optical sections through the $z$ axis. Similar results were obtained in two additional experiments. $\boldsymbol{B}$, Confocal micrograph of a midbody ganglion (anterior up) where one CV neuron was loaded with Neurobiotin and a VI-2 neuron was loaded with rhodamine-conjugated dextran; the image results from the maximal intensity projections of $691 \mu$ m optical sections through the $z$ axis. Similar results were obtained in two additional experiments. $C$, Confocal micrograph of a midbody ganglion (anterior up) where one CV neuron was loaded with Neurobiotin and an NS neuron was loaded with rhodamine-conjugated dextran; the image results from the maximal intensity projections of $851 \mu \mathrm{m}$ optical sections through the $z$ axis. Similar results were obtained in three additional experiments. $\boldsymbol{A}-\boldsymbol{C}$, White triangles represent at the $\mathrm{CV}$ neuron that was loaded with Neurobiotin. White bar represents a scale of $100 \mu \mathrm{m}$.

\section{$\mathrm{CV}$ resets the crawling motor pattern}

To evaluate whether, in addition to controlling the circular muscle fibers, CV plays a role in the circuit that controls crawling, its membrane potential was manipulated in the course of crawling episodes. Brief (compared with the cycle period) excitatory pulses were injected in CV neurons at different phases of the crawling cycle (phase angle) and their effect on crawling was evaluated by analyzing the rhythmic activity of DE-3. The rationale that guided this set of experiments was that, if $\mathrm{CV}$ was a mere output element, manipulation of its membrane potential should not affect the rhythmic activity of DE-3; if CV was connected (directly or indirectly) to DE-3, the rhythmic activity of the latter should be temporarily altered, but beyond this perturbation lapse the rhythm should resume according to the ongoing input from the unaffected pattern generator; if, instead, CV was connected to the pattern generator, manipulation of its membrane potential should reset the rhythmic activity observed in DE-3 (Friesen et al., 1976).

Pulses that prolonged the firing activity of CV, before a DE-3 burst was initiated, caused an increase in the period or phase delay (Fig. 6A). The same stimulus applied during the DE-3 burst interrupted it, leading to a new burst at the end of the pulse (Fig. $6 B$ ) and, provided that the pulse duration was shorter than the period, it caused a shortening of the cycle or phase advance. Figure $6 \mathrm{C}$ summarizes the results obtained in experiments where we used pulses that lasted 2, 3.5, and $6 \mathrm{~s}$, in episodes whose average period was $\sim 6 \mathrm{~s}$. The graph shows that the period change produced by short excitatory pulses in $\mathrm{CV}$ was a linear function of the phase angle at which the pulse was applied. The degree to which the phase was advanced or delayed depended on the duration of the pulse. As expected, pulses whose duration was similar to the cycle period caused only phase delays.

Although our results indicate that a direct electrical interaction between $\mathrm{CV}$ and the inhibitors of longitudinal muscles is unlikely (see analysis of the results shown in Fig. $4 B$ ), a chemically mediated inhibitory pathway between CV and DE-3 is still possible. This inhibitory interaction could explain that excitation of CV stops DE-3 firing, but it could not explain the observed phase shift. Without a connection to the pattern generator, no phase shift would be expected, but a transient interruption of DE-3 firing, after which the ongoing rhythm should have been re- vealed. A putative direct electrical or chemical excitatory connection between CV and DE-3 could not account for the results, as what we observed is that excitation of CV prevents the activity of DE-3.

Thus, in our view, the results strongly suggest the existence of a retrograde link from $\mathrm{CV}$ motoneurons to the neurons that form the pattern generator circuit.

\section{$\mathrm{CV}$ activity influences the crawling period}

$\mathrm{CV}$ could be an intrinsic part of the pattern generator or, alternatively, participate as an extrinsic modulator. To distinguish between these two alternatives, we evaluated the effect of "functionally removing" CV from the circuit by hyperpolarizing it in the course of a crawling episode. If CV was an intrinsic element of the oscillator, its hyperpolarization should disrupt the rhythm, whereas if the feedback had a modulatory function, the rhythm should persist but run with, for example, a different period.

Negative $4 \mathrm{nA}$ pulses were injected in one CV neuron spanning several cycles of dopamine-induced crawling episodes (Fig. $7 A$ ). Hyperpolarizing a CV neuron produced a small but reliable reduction of the cycle period (Fig. $7 \mathrm{Bi}$ ), but a more revealing description of the effect of CV hyperpolarization was obtained by plotting the relative cycle period (the ratio between the period during the pulse over that previous to the pulse) as a function of the period preceding the current injection (Fig. 7Bii). This graph shows that the degree of the pulse effect exhibited a negative correlation with the cycle period. In other words, the longer the period, the larger the relative acceleration evoked by CV hyperpolarization.

Given the period change, we analyzed the effect of CV hyperpolarization on the duty cycle of DE-3 rather than examining the burst duration per se. This variable was not affected by the change in CV membrane potential (Fig. 7C; $p=0.21$, Wilcoxon Rank Sign Test for paired data), whereas the firing frequency of DE-3 was decreased by $\sim 15 \%$ when $\mathrm{CV}$ was hyperpolarized (Fig. $7 D$ ).

Because the two CV motoneurons appear as electrically independent of one another (Fig. 4A), it was possible that inhibition of one CV was not enough to disrupt the rhythmic pattern because the contralateral homolog was sufficient to maintain it. To analyze this possibility, we compared the effect of injecting negative $4 \mathrm{nA}$ in one $\mathrm{CV}$ neuron (individual) versus injection in both 
A
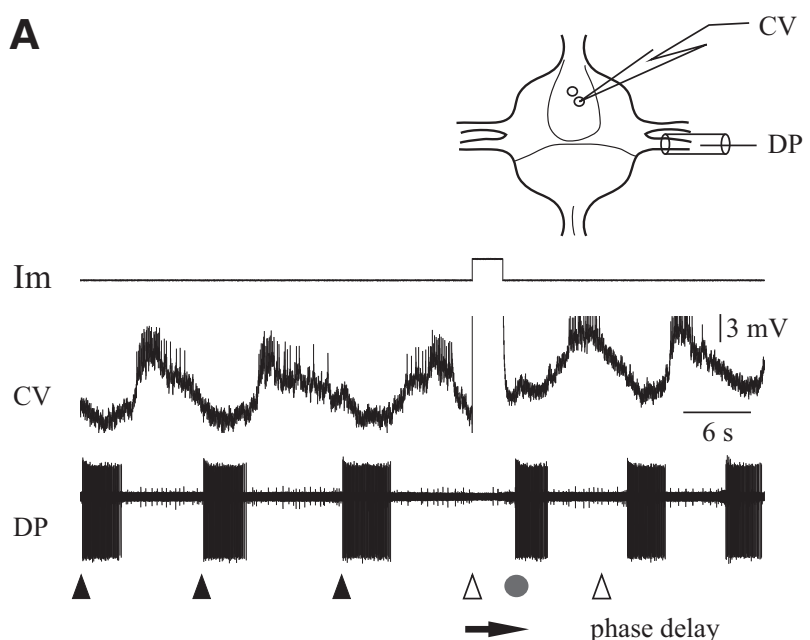

B

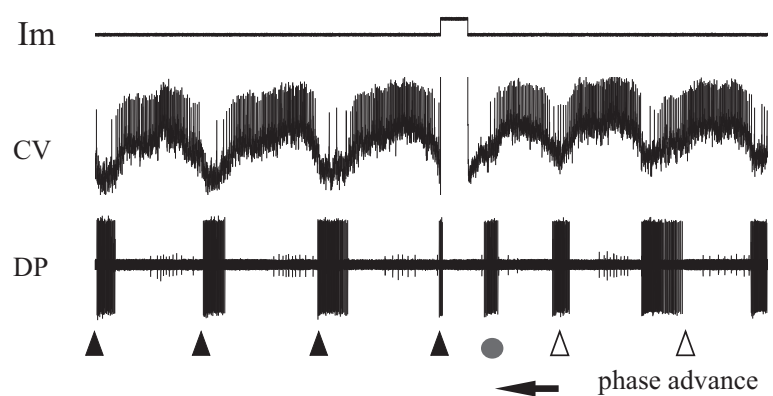

C

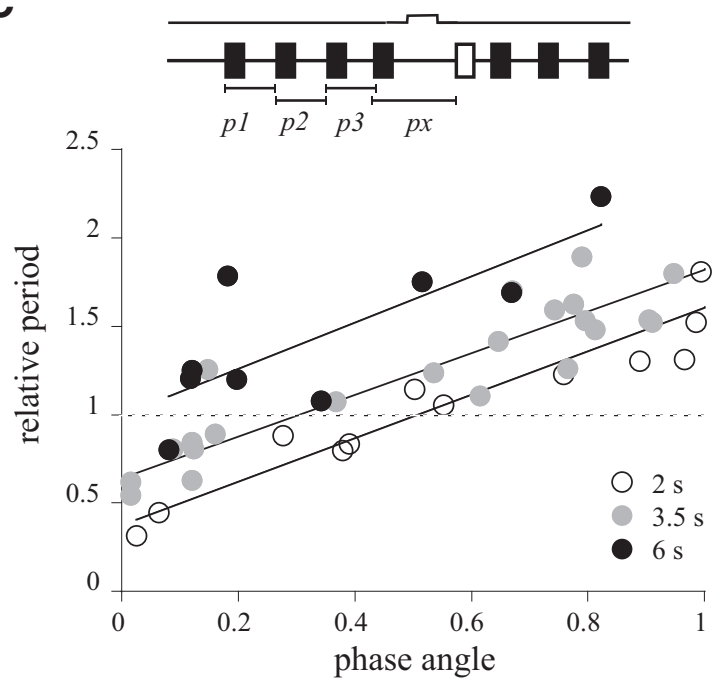

Figure 6. Activation of $\mathrm{CV}$ motoneuron resets the crawling motor pattern. A, Diagram represents the recording configuration: intracellular recording of a CV motoneuron and extracellular recording of DE-3 in a DP nerve. A current pulse was injected in CV motoneuron (Im) that prolonged its firing activity during one cycle a crawling episode. Black triangles underneath the $D P$ recording represent the start of the DE- 3 bursts preceding the intervention. White triangle represents the approximate timing where the two next DE-3 bursts were expected if the intervention had no effect on the cycle period. Gray dot represents the actual beginning of the DE-3 burst following the intervention, indicating a delay in the motoneuron firing. $\boldsymbol{B}$, Same as in $\boldsymbol{A}$, but the pulse was injected during the DE-3 burst, interrupting it. DE-3 started firing a burst after the end of the CV pulse, which happened (gray dot) before the expected period (white triangle), revealing a phase advance. $\boldsymbol{C}$, Top, Schematic representation of the analysis: previous to the current pulse, three periods $(p 1, p 2$, and $p 3)$ were measured and an average was calculated $(P)$; the effect of the pulse was expressed as a relative period calculated as $p x / P$. Graph represents the relative period as a function of the timing of the injection within the corresponding cycle
A

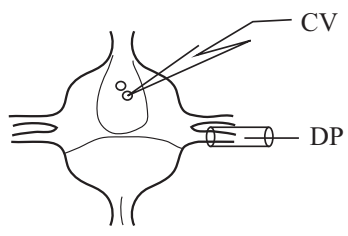

Im

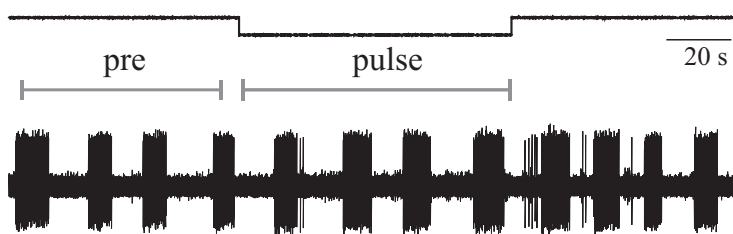

Bi

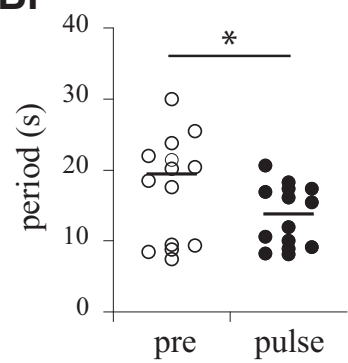

$\mathrm{Bii}$

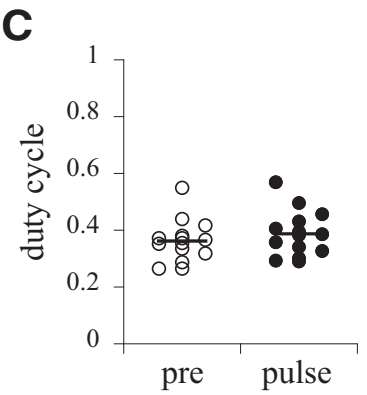

D

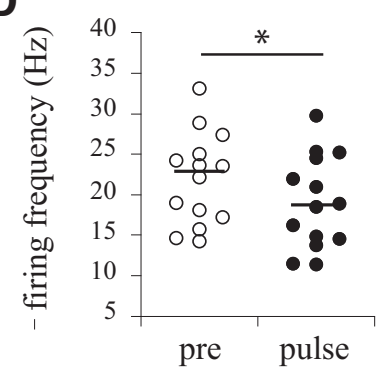

Figure 7. Inhibition of $\mathrm{CV}$ during crawling disrupts the motor pattern. $A$, Diagram represents the recording configuration: extracellular recording of a DP nerve during a crawling episode as a CV neuron (recording not shown) was subjected to a negative $4 \mathrm{nA}$ pulse indicated by the step in the top line (Im). Bi, Cycle period measured before (pre) and during the pulse. ${ }^{*} p=0.02$ (Wilcoxon Rank Sign Test for paired data, $n=14$ ). Bii, Graph represents the ratio between the cycle period during the pulse and the cycle period previous to the pulse (relative period) as a function of the latter for the protocol shown in $\boldsymbol{A}$. Line indicates the linear fit $(r=0.77, p=$ 0.00048 ). C, Duty cycle of DE-3 bursts before (pre) and during the pulse. $D$, Firing frequency of DE-3 before (pre) and during the pulse. ${ }^{*} p=0.00085$ (Wilcoxon Rank Sign Test for paired data, $n=14)$.

CVs simultaneously (dual) on the cycle period. On average, the relative period (period during the pulse(s) over period before the pulse(s) $)$ in individual and dual tests $(0.84 \pm 0.06$ and $0.74 \pm 0.1$, respectively; $n=14$ ) were not significantly different ( $p=0.17$, Wilcoxon test for paired data).

Because inhibition of CV motoneurons did not disrupt the rhythmic output, we conclude that CV is not an intrinsic com-

$\leftarrow$

(phase angle). Different symbols discriminate for current pulses $(2-4 \mathrm{nA})$ of different duration, and the lines indicate linear fits: $r=0.95, r=0.89$, and $r=0.78$ for pulses of $2.0(n=18$ pulses, 6 ganglia, 3 animals), 3.5 ( $n=26$ pulses, 6 ganglia, 2 animals), and $6.0 \mathrm{~s}$ ( $n=9$ pulses, 4 ganglia, 2 animals), respectively ( $p=0.000001, p=0.000001$, and $p=0.01$, respectively). The average $( \pm S D)$ period of the crawling segments in this series of experiments was $5.3 \pm 2$, $5.3 \pm 1.4$, and $6.1 \pm 2.4 \mathrm{~s}$ for the experiments subjected to pulses of $2.0,3.5$, and $6.0 \mathrm{~s}$, respectively. 
ponent of the oscillatory network but linked to it by a feedback connection. Because inhibition of CV shortened the period in proportion to its control value, we interpret that CV exerts a positive feedback on the unit(s) of the pattern generator that controls the elongation phase. According to this interpretation, the larger the weight of this feedback, the longer the elongation phase and the bigger the effect of CV "removal" from the circuit.

It is worth noting that, because each CV was coupled to another nine neurons in the ganglion, the effect could be mediated through any of these electrically coupled neurons. Among them, the NS cells are unlikely to be involved because the motoneuron-NS junctions rectify and are not conducting when the motoneuron membrane potential is more negative than that of NS (Rela and Szczupak, 2007).

\section{Each CV neuron exerts symmetrical effects}

During crawling, the animal displays bilaterally symmetrical movements and this right-left symmetry is also observed at the level of the isolated ganglion: when both DP nerves are recorded, the DE-3 bursts occurred simultaneously (Fig. 8). We thus examined the effect of manipulating the membrane potential of one CV on the activity of both DP nerves in the course of dopamineinduced crawling. Figure $8 A$ illustrates that the phase shift caused by short excitatory pulses affects simultaneously both DP nerves, whereas Figure $8 B$ illustrates that hyperpolarization of CV did not disrupt the symmetrical activity. These experiments suggest that the pattern generator to which CV sends feedback signals has symmetrical motor output.

\section{Discussion}

\section{CV motoneurons send feedback signals to the} pattern generator

The CV motoneurons innervate the ventral circular muscles that provide to the elongation phase of crawling. In dopamineinduced crawling, the activity of these motoneurons spanned a preponderant part of the cycle, covering $\sim 60 \%$ of the period, whereas the activity of DE-3, which provides to the contraction phase, was limited to $\sim 30 \%$ of the period (Fig. 2). This picture, obtained in the isolated ganglion, matches that obtained in behavioral studies (Stern-Tomlinson et al., 1986; Cacciatore et al., 2000). Together, these findings indicate that the phase relationship is, to a large extent, controlled by the pattern generator located in each individual ganglion of the CNS.

In addition to their action on circular muscles, the experiments described here revealed that $\mathrm{CV}$ motoneurons were connected to the network that controls the rhythmic pattern: experimental excitation of CV neurons during crawling reset the rhythmic motor pattern (Fig. 6), and inhibition of the motoneuron caused a period-dependent acceleration (Fig. 7). These data were interpreted according to the model depicted in Figure 9: at the level of the individual ganglia, crawling is controlled by a "core" pattern generator (cPG; presumably one in each ganglion) formed by contraction (C) and elongation (E) units, that regulate the activity of the motoneurons that activate longitudinal (DE-3) and ventral circular $(\mathrm{CV})$ muscles, respectively. In the absence of additional information, and in agreement with previous assessments (Baader and Kristan, 1995; Cacciatore et al., 2000; Esch et al., 2002), the cPG was conceived as a single half-center oscillator that controls the alternation between elongation and contraction.

Based on the reset experiments (Fig. 6), where excitation of the motoneuron caused changes in the cycle compatible with excitation of $\mathrm{E}$, the model proposes that $\mathrm{CV}$ is connected to $\mathrm{E}$ via an excitatory connection. Perturbation of one CV neuron pro-
A

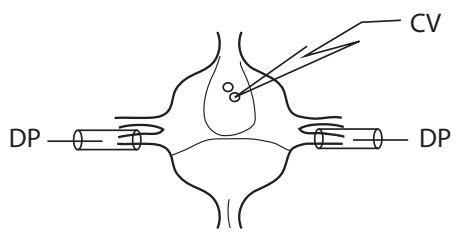

$\mathrm{Im}$

DP1

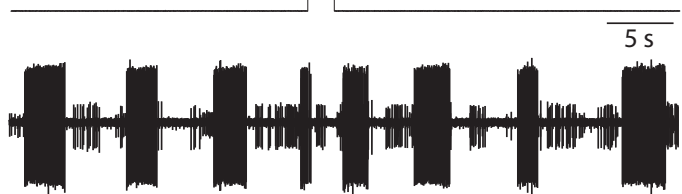

DP2

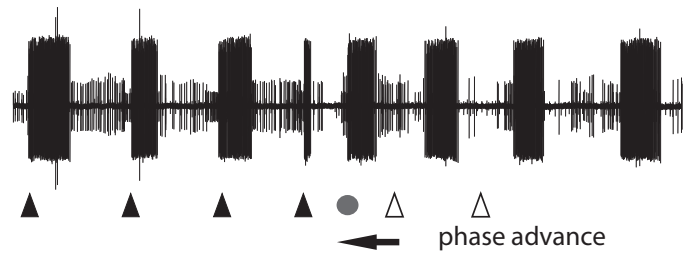

B

$\mathrm{Im}$

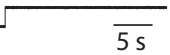

DP1

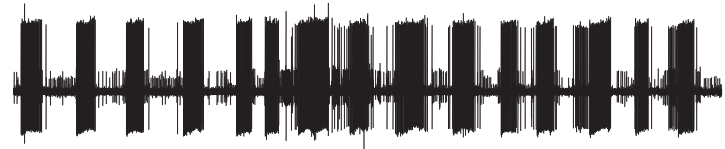

DP2

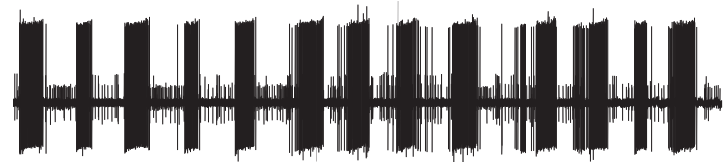

Figure 8. Manipulation of CV membrane potential exerts a symmetrical effect. $\boldsymbol{A}$, Diagram represents the recording configuration: extracellular recordings of both DP nerves and intracellular recording of CV. During a crawling episode, the CV neuron (recording not shown) was subjected to a positive $4 \mathrm{nA}$ pulse indicated by the step in the top line (Im). There is coincident change in activity in both nerves. Black triangles beneath the DP recording represent the start of the DE-3 bursts preceding the pulse step. White triangle represents the approximate timing where the two next DE-3 bursts were expected if the pulse step had no effect on the period of the rhythmic activity. Gray dot represents the actual beginning of the DE-3 burst following the pulse step, indicating a delay in the motoneuron firing. Similar results were observed in additional 4 experiments performed in 2 crawling episodes. $\boldsymbol{B}$, Same as in $\boldsymbol{A}$, but a CV neuron was subjected to a negative $4 \mathrm{nA}$ pulse. Similar results were observed in additional 10 experiments performed in 5 crawling episodes.

duced symmetrical left-right effects; therefore, we assumed that the $\mathrm{CPG}$ exerts a symmetrical left-right control.

The dye-coupling experiments offered a first view on the connectivity of CV motoneurons. The connectivity obtained is restricted to electrical synapses and because these connections could rectify the picture obtained may be limited to the transmission of excitatory signals from CV to the coupled neurons. Future work should establish the role that these coupled neurons play in crawling.

\section{Putative functional role of $\mathrm{CV}$ feedback connection}

The present report is a clear demonstration that motoneurons do not only serve as output units but are integral elements of the networks that control rhythmic behavior due to feedback connections with the circuit that controls the motor pattern. Although proprioceptive feedback from the muscles that motoneurons innervate play well-documented physiological roles (Hultborn, 2006), feedback from the motoneurons themselves 


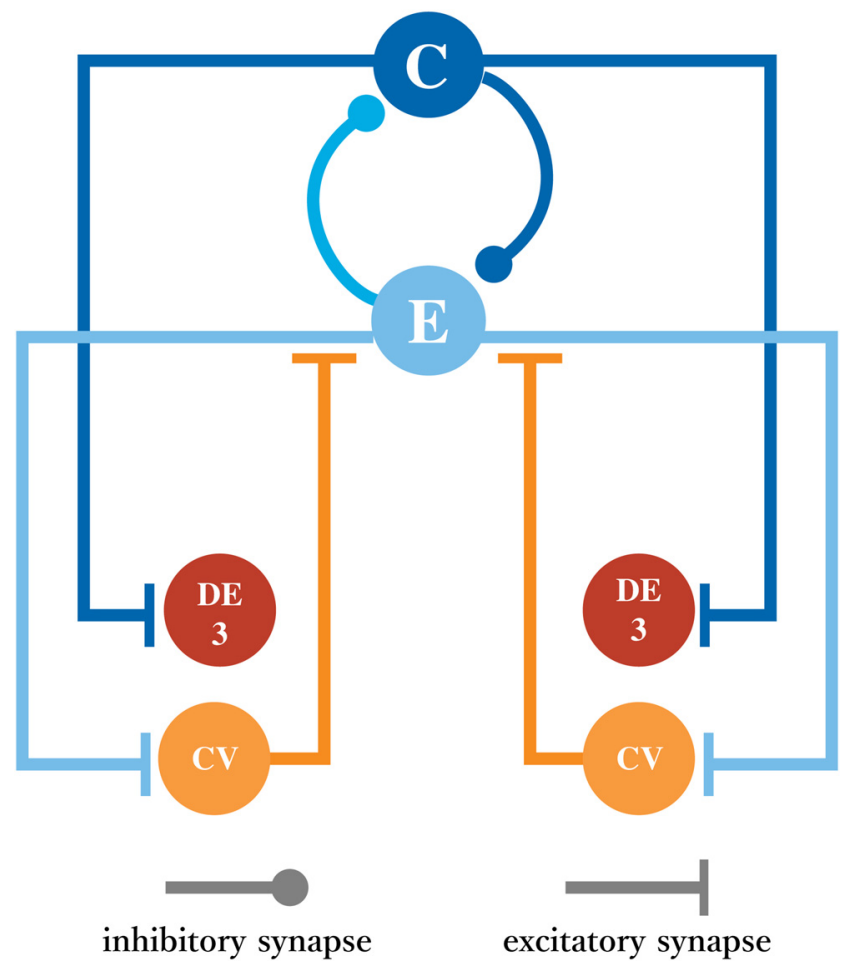

Figure 9. Proposed network interactions between the CV motoneuron and the crawling pattern generator. Two units $(C, E)$ constitute the pattern generator, forming a half-center oscillator that controls left and right motoneurons. The $C$ unit excites the bilateral pair of DE-3 motoneurons, and the E unit excites the bilateral pair of CV motoneurons. According to the results (Figs. 6, 7) we propose that $\mathrm{CV}$ motoneurons feed back excitation onto $\mathrm{E}$. It is important to notice that DE-3 is representative of another 7 excitors of longitudinal fibers (Ort et al., 1974), and $\mathrm{CV}$ is representative of at least another excitor of circular muscles (Stuart, 1970).

adds a level of control whose role is presently under active investigation (Nishimaru et al., 2006; Szczupak, 2014; Moore et al., 2015; Song et al., 2016; Matsunaga et al., 2017).

We hypothesize that, in the leech crawling network, the positive feedback from $\mathrm{CV}$ motoneuron to the $\mathrm{CPG}$ regulates the elongation-contraction balance. In other words, this connectivity could be partly responsible for the dominance of the elongation phase.

To test this specific hypothesis, we built a simple computational model, based on the connectivity pattern suggested in Figure 9. The aim of the model was testing the role of the positive feedback from one output unit to the oscillator in an otherwise balanced network (no phase dominance). The CPG was represented by two identical units (E and C) using the Morris-Lecar model (see Materials and Methods). The two units were linked by mutually inhibitory connections of equal strength. The motoneurons CV and DE-3 were modeled as two identical passive units (i.e., they did not exhibit any voltage-dependent conductance), that receive identical synaptic excitation from $\mathrm{E}$ and $\mathrm{C}$, respectively. To test the role of the positive feedback, the circuit was tested in the absence and presence of an excitatory projection from CV to E. We emphasize that this model does not intend to describe quantitative details of the crawling circuit but to examine the posed hypothesis. The traces represent the membrane potential envelope displayed by the neurons during the rhythmic activity; action potentials were not included in the model.

Figure $10 \mathrm{~A}$ shows that application of identical basal excitatory current (bias current) to units $\mathrm{E}$ and $\mathrm{C}$ engaged the network in an

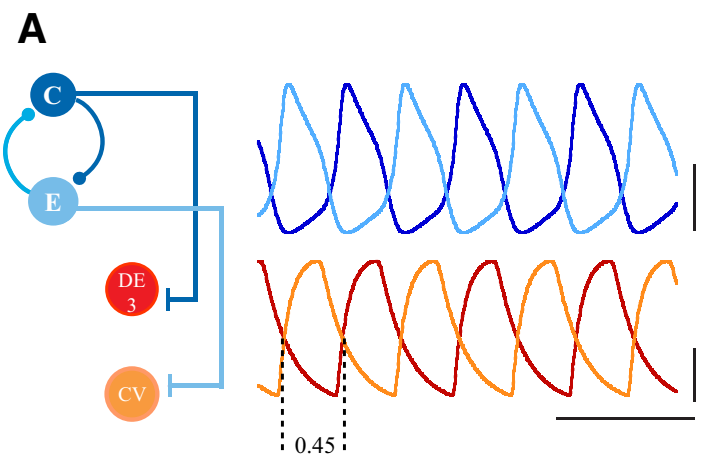

B
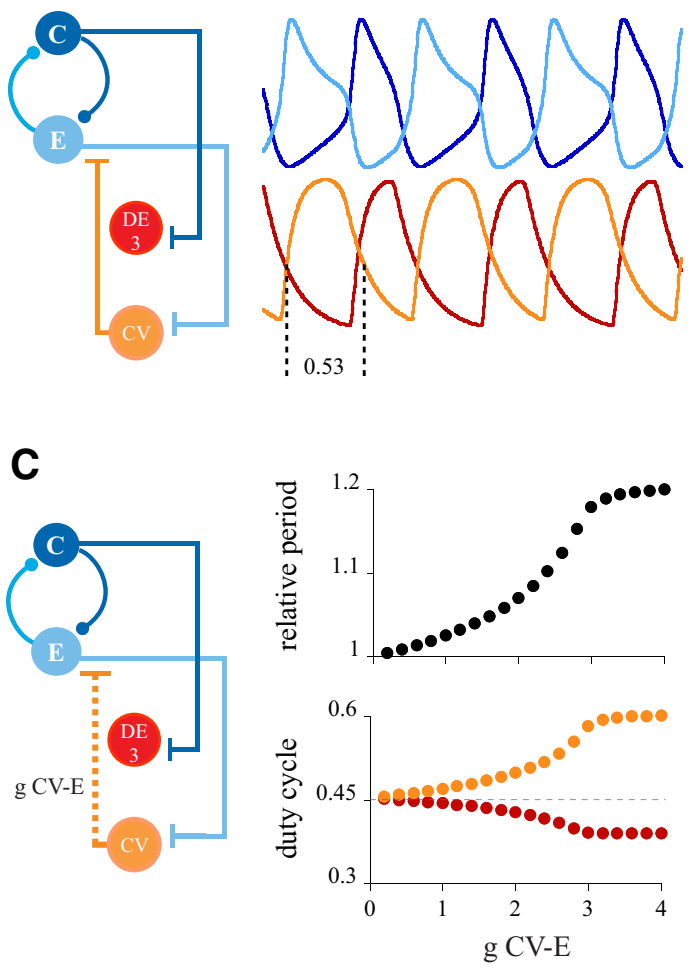

Figure 10. A computational model tested the feedback effect. $\boldsymbol{A}$, Left, Diagram represents the connectivity diagram of the model. Cells $E$ and C were modeled as Morris-Lecar units interconnected by mutually inhibitory chemical synapses. The motoneurons DE-3 and CV were modeled as passive units (do not exhibit voltage-dependent conductances). C is connected to $D E-3$ and $E$ to $C V$ via excitatory chemical synapses. Right, Top traces represent the resulting rhythmic electrical activity of $C$ and $E$. Bottom traces represent the activity that this C-E alternation imposes on the motoneurons, following the color code of the diagram. Dotted lines indicate the duty cycle of CV (measured as the halfwidth of the wave) and the number its value. Calibration: Vertical, $20 \mathrm{mV}$; Horizontal, $10 \mathrm{~s}$. $\boldsymbol{B}$, Same as in $\boldsymbol{A}$, but including an excitatory feedback from $C V$ to $E$ via a chemical synapse. $\boldsymbol{A}, \boldsymbol{B}$, Plotted in the same voltage and time scale (vertical scales are 40 and $20 \mathrm{mV}$ for ( and E, respectively). Calibration: Horizontal, 10 s. C, Diagram represents that the CV-E conductance ( $\mathrm{g}$ CV-E) was varied. Top, Relationship between the cycle period and the maximal g CV-E conductance (relative to the period at $g(V-E=0)$. Bottom, Duty cycle of DE-3 (red) and CV (orange) as a function of $g$ CV-E.

oscillatory activity that led them to generate out-of-phase waves of potential that, in turn, drove CV and DE-3. In the absence of any feedback connection, the cycle period was $8.4 \mathrm{~s}$ and the duty cycles of CV and DE-3 were identical (0.45). Figure $10 B$ shows the same circuit, with the addition of a positive feedback from the $\mathrm{CV}$ to $\mathrm{E}$. This addition increased the period to $9.4 \mathrm{~s}$ and the duty cycle of CV to 0.53 , whereas the duty cycle of DE-3 was reduced to 0.41 . It is noteworthy that the duty cycle of DE-3 was reduced because 
the period was increased, but its half-width remained the same whereas that of CV increased.

Physiological experiments tested the influence of $\mathrm{CV}$ on the rhythmic activity by applying hyperpolarizing pulses in $\mathrm{CV}$ in the course of crawling episodes (Fig. 7). CV hyperpolarization accelerated the rhythm, and the extent of this effect depended on the magnitude of the control period (before the pulse; Fig. $7 B$ ). A plausible interpretation of this result states that the hyperpolarization "removed" CV from the circuit, and this maneuver influenced the period in proportion to the influence that $\mathrm{CV}$ exerted on the period in any given preparation; or in other words, the acceleration was proportional to the strength of the feedback connection.

To test this interpretation in the model, we varied the maximal synaptic conductance of the CV-E connection and examined its influence on the circuit output. Figure $10 \mathrm{C}$ shows that, as the feedback conductance was increased, the period of crawling was increased. The duty cycle of CV paralleled the increase in the period, whereas the duty cycle of DE-3 decreased, although to a smaller degree than the $\mathrm{CV}$ increase. The latter results contradict the experimental data that indicated that DE-3 duty cycle was unaffected by $\mathrm{CV}$ hyperpolarization (Fig. $7 \mathrm{C}$ ), indicating that the model misses a mechanism to control the stability of the contraction phase.

Despite this one discrepancy, the model supports the hypothesis that the dominance of elongation in crawling could be caused by the connectivity of $\mathrm{CV}$ on the rhythm generator. The proposed mechanism does not rule out other factors to control phase duration, such as differential background excitation of the neurons that form the pattern generator circuit, differential intrinsic ionic properties of these neurons, negative feedback from mechanosensory inputs, or different gains in their connections to the motoneurons (Yakovenko et al., 2005; Grillner, 2006; Büschges et al., 2008; Spardy et al., 2011), but uncovers a novel mechanism that enriches the repertoire of functional roles played by feedback mechanisms in general, and of motoneuron feedback in particular.

The data presented here establish a possible clear role for the feedback from motoneurons to the CNS. Studies performed in the leech nervous system offer the advantage of studying the role of specific neurons with well-delimited functions; and in the present case, it allowed to recognize that the prominence of the elongation phase of crawling was a function of the feedback between the motoneurons active during this particular phase and the pattern generator.

\section{References}

Baader AP, Kristan WB (1995) Parallel pathways coordinate crawling in the medicinal leech, Hirudo medicinalis. J Comp Physiol A 176:715-726. Medline

Barth FG (2000) How to catch the wind: spider hairs specialized for sensing the movement of air. Naturwissenschaften 87:51-58. CrossRef Medline

Büschges A, Akay T, Gabriel JP, Schmidt J (2008) Organizing network action for locomotion: insights from studying insect walking. Brain Res Rev 57:162-171. CrossRef Medline

Cacciatore TW, Rozenshteyn R, Kristan WB Jr (2000) Kinematics and modeling of leech crawling: evidence for an oscillatory behavior produced by propagating waves of excitation. J Neurosci 20:1643-1655. Medline

Drew T, Marigold DS (2015) Taking the next step: cortical contributions to the control of locomotion. Curr Opin Neurobiol 33:25-33. CrossRef Medline

Eisenhart FJ, Cacciatore TW, Kristan WB (2000) A central pattern genera- tor underlies crawling in the medicinal leech. J Comp Physiol A Neuroethol Sens Neural Behav Physiol 186:631-643. CrossRef Medline

Esch T, Mesce KA, Kristan WB (2002) Evidence for sequential decision making in the medicinal leech. J Neurosci 22:11045-11054. Medline

Fan RJ, Marin-Burgin A, French KA, Otto Friesen W (2005) A dye mixture (Neurobiotin and Alexa 488) reveals extensive dye-coupling among neurons in leeches: physiology confirms the connections. J Comp Physiol A Neuroethol Sens Neural Behav Physiol 191:1157-1171. CrossRef Medline

Friesen WO, Poon M, Stent GS (1976) An oscillatory neuronal circuit generating a locomotory rhythm. Proc Natl Acad Sci U S A 73:3734-3738. CrossRef Medline

Friesen WO, Poon M, Stent GS (1978) Neuronal control of swimming in the medicinal leech: IV. Identification of a network of oscillatory interneurones. J Exp Biol 75:25-43. Medline

Georgopoulos AP, Carpenter AF (2015) Coding of movements in the motor cortex. Curr Opin Neurobiol 33:34-39. CrossRef Medline

Goulding M (2009) Circuits controlling vertebrate locomotion: moving in a new direction. Nat Rev Neurosci 10:507-518. CrossRef Medline

Gray J, Lissmann HW, Pumphrey RJ (1938) The mechanism of locomotion in the leech (Hirudo medicinalis Ray). J Exp Biol 15:408-430.

Grillner S (2006) Biological pattern generation: the cellular and computational logic of networks in motion. Neuron 52:751-766. CrossRef Medline

Hultborn H (2006) Spinal reflexes, mechanisms and concepts: from Eccles to Lundberg and beyond. Prog Neurobiol 78:215-232. CrossRef Medline

Kristan WB Jr, Calabrese RL (1976) Rhythmic swimming activity in neurones of the isolated nerve cord of the leech. J Exp Biol 65:643-668. Medline

Kristan WB Jr, Calabrese RL, Friesen WO (2005) Neuronal control of leech behavior. Prog Neurobiol 76:279-327. CrossRef Medline

Matsunaga T, Kohsaka H, Nose A (2017) Gap junction-mediated signaling from motor neurons regulates motor generation in the central circuits of larval Drosophila. J Neurosci 37:2045-2060. CrossRef Medline

Maynard DM, Selverston AI (1975) Organization of the stomatogastric ganglion of the spiny lobster. J Comp Physiol A Neuroethol Sens Neural Behav Physiol 100:161-182. CrossRef

Moore NJ, Bhumbra GS, Foster JD, Beato M (2015) Synaptic connectivity between Renshaw cells and motoneurons in the recurrent inhibitory circuit of the spinal cord. J Neurosci 35:13673-13686. CrossRef Medline

Morris C, Lecar H (1981) Voltage oscillations in the barnacle giant muscle fiber. Biophys J 35:193-213. CrossRef Medline

Muller KJ, Nicholls JG, Stent GS (1981) Neurobiology of the leech. Cold Spring Harbor, NY: Cold Spring Harbor Laboratory.

Nishimaru H, Restrepo CE, Kiehn O (2006) Activity of Renshaw cells during locomotor-like rhythmic activity in the isolated spinal cord of neonatal mice. J Neurosci 26:5320-5328. CrossRef Medline

Ort CA, Kristan WB, Stent GS (1974) Neuronal control of swimming in the medicinal leech. J Comp Physiol A Neuroethol Sens Neural Behav Physiol 94:121-154. CrossRef

Bernardo Perez-Etchegoyen C, Alvarez RJ, Rodriguez MJ, Szczupak L (2012) The activity of leech motoneurons during motor patterns is regulated by intrinsic properties and synaptic inputs. J Comp Physiol A Neuroethol Sens Neural Behav Physiol 198:239-251. CrossRef Medline

Poon M, Friesen WO, Stent GS (1978) Neuronal control of swimming in the medicinal leech: V. Connexions between the oscillatory interneurones and the motor neurones. J Exp Biol 75:45-63. Medline

Puhl JG, Mesce KA (2008) Dopamine activates the motor pattern for crawling in the medicinal leech. J Neurosci 28:4192-4200. CrossRef Medline

Puhl JG, Masino MA, Mesce KA (2012) Necessary, sufficient and permissive: a single locomotor command neuron important for intersegmental coordination. J Neurosci 32:17646-17657. CrossRef Medline

Rela L, Szczupak L (2007) In situ characterization of a rectifying electrical junction. J Neurophysiol 97:1405-1412. Medline

Rinzel J, Ermentrout B (1998) Analysis of neural excitability and oscillations. In: Methods in neural modeling (Koch C, Segev I, eds), pp 251-292. Cambridge, MA: Massachusetts Institute of Technology.

Selverston AI, Moulins M (1985) Oscillatory neural networks. Annu Rev Physiol 47:29-48. CrossRef Medline 
Song J, Ampatzis K, Björnfors ER, El Manira A (2016) Motor neurons control locomotor circuit function retrogradely via gap junctions. Nature 529:399-402. CrossRef Medline

Spardy LE, Markin SN, Shevtsova NA, Prilutsky BI, Rybak IA, Rubin JE (2011) A dynamical systems analysis of afferent control in a neuromechanical model of locomotion: I. Rhythm generation. J Neural Eng 8:065003. CrossRef Medline

Staras K, Kemenes G, Benjamin PR (1998) Pattern-generating role for motoneurons in a rhythmically active neuronal network. J Neurosci 18: 3669-3688. Medline

Stern-Tomlinson W, Nusbaum MP, Perez LE, Kristan WB (1986) A kinematic study of crawling behavior in the leech, Hirudo medicinalis. J Comp Physiol A 158:593-603. CrossRef Medline
Stuart AE (1970) Physiological and morphological properties of motoneurones in the central nervous system of the leech. J Physiol (Lond) 209:627646. CrossRef Medline

Szczupak L (2014) Recurrent inhibition in motor systems, a comparative analysis. J Physiol Paris 108:148-154. CrossRef Medline

Wadepuhl M (1989) Depression of excitatory motoneurones by a single neurone in the leech central nervous system. J Exp Biol 143:509-527. Medline

Wagenaar DA (2015) A classic model animal in the 21st century: recent lessons from the leech nervous system. J Exp Biol 218:3353-3359. CrossRef Medline

Yakovenko S, McCrea DA, Stecina K, Prochazka A (2005) Control of locomotor cycle durations. J Neurophysiol 94:1057-1065. CrossRef Medline 